\title{
Comment in Reply to "On the complexity of extending the convergence region for Traub's method"
}

Andy E. Williams, Nobeah Foundation, Nairobi, Kenya, awilliams@nobeahfoundation.org

This comment is in reply to the paper "On the complexity of extending the convergence region for Traub's method" [1]. In complexity science, from the mathematical perspective, discussions of complexity often concern algorithmic complexity such as in the paper responded to here [2]. But this is only one of the kinds of complexity that exists even in the mathematical domain. There is also the complexity in the behavior of a system of equations; there is the complexity of the reasoning or algorithm required to understand a system of equations ("understand" interpreted here as defining the problem needing to be solved); and, as mentioned, there is the complexity of the reasoning or algorithm required to solve a system of equations.

The newly emerging science of Human-Centric Functional Modeling [3] suggests a definition of complexity that is hypothesized to apply in all possible cases in mathematics as well as in all systems outside mathematics as well. In Human-Centric Functional Modeling, the functions of any system are observed from the human perspective as taking the system to different functional states, so that the system moves through a space of functional states, or a "functional state space". From this perspective the human cognitive system (the "mind") moves from one concept (one functional state) that might be a collection of concepts, to another concept (another functional state), and in so doing moves through the functional state space of the mind (a space of concepts or a "conceptual space").

Every functional state space for any system can potentially be represented the same way as this conceptual space, that is, in terms of a graph containing a network of nodes (each of which in the case of the cognitive system represent concepts), where the edges connecting those nodes represent the processes through which those nodes interact within the system (which in the case of the cognitive system are the reasoning processes through which concepts interact). Furthermore, every system can potentially be represented as a network in this way [7]. Since the definition of the complexity of reasoning, or of any algorithm automating that reasoning has been hypothesized in the conceptual space to be the length of the reasoning in conceptual space multiplied by the linear density of concepts that the reasoning must move through, then since all other systems can potentially be represented in terms of functional state spaces, this definition of complexity is hypothesized to apply to all other systems as well.

Just as Human-Centric Functional Modeling represents the cognition as moving through a space of concepts, it also represents the consciousness as moving through a space of awareness's in which complexity is defined in the same way. This awareness space is important from a mathematical point of view because aside from the processes that can be conceptualized as reasoning, and therefore that might be automated in algorithms, there are also processes that potentially cannot be conceptualized but that the consciousness can be aware of. For example, this awareness space of the consciousness can contain awareness's of sensory perceptions, emotions, and perhaps other entities that might not be able to be conceptualized. In this sense an awareness is a more abstract category, a more abstract representation of information. If "truth" can in some way be represented as having levels of abstraction, then the truth in an awareness state must somehow have the capacity to contain the truth in a sensory state and therefore in comparison to the truth in a sensory state must in some way be more general. 
Defining some representation of truth is important to our understanding of complexity, though this has been a question mathematics and philosophy have struggled with for some time [5].

Truth can be defined in two ways. Relative truth is a signal. Any given reasoning processes in conceptual space, or any given process in any other functional state space, is a channel that conveys truth with some degree of error that is given by information theory. In other words, in conceptual space the relative truth of one claim can be defined in terms of the self-consistency of the group of other claims contained in the generalization of that claim. In addition, any geometry in any Euclidean or positively or negatively curved space is hypothesized here to represent an absolute truth, but whether all truths can be represented by some geometry has not been proved. In addition, any geometry in any Euclidean or positively or negatively curved space is hypothesized here to represent an absolute truth, but whether all truths can be represented by some geometry has not been proved.

Assume that in conceptual space statement A is truthful (full of truth B), but that all of truth B does not fit in statement A. What representation of truth B is consistent with this assumption? Assuming that the human cognitive system moves through a graph containing a network of concepts connected by reasoning processes, which we will call the "conceptual space", then if $\mathrm{C}$ is the set of all statements that are full of truth B, and if each statement in $\mathrm{C}$ forms a path in conceptual space, then $\mathrm{C}$ is some subset of a geometry in conceptual space. Furthermore, for any geometry D in conceptual space, there is a set of statements that are full of the truth of $\mathrm{D}$, and therefore a set of statements that are truthful, which define that geometry. If the awareness space might contain a greater subset of truths than the conceptual space, then it is also true of the awareness space that for any geometry D in awareness space, there is a set of statements that are full of the truth of D.

In conceptual space, as in any other functional state space, truth might be considered to be a signal transmitted by a reasoning process (which in other functional state spaces in general represents an interaction process) [8]. The capacity of any channel to transmit a signal is determined by information theory. One expression of Information Theory is the Shannon-Hartley theorem [4] which states that the channel capacity $\mathrm{C}$ is given by:

$C=B \times \log _{2}\left(1+\frac{S}{N}\right)$

In the physical state space representing matter and energy, these quantities: B the bandwidth of the channel; $\mathrm{N}$ the average power of the noise and interference over the bandwidth; and $\mathrm{S} / \mathrm{N}$ the signal-tonoise ratio, have all been determined. These quantities remain to be interpreted and the equation remains to be validated in the conceptual space.

In general, for any geometry D in any space (whether Euclidean, or whether positively or negatively curved) for any $\mathrm{N}$ dimensions, assume that the set of all paths belonging to that geometry are statements full of the truth of that geometry and are therefore truthful. The question is can it be shown that in general any truth $\mathrm{D}$ is a geometry in some $\mathrm{N}$ dimensional space, and that all geometries in any space are a representation of some truth D?

Part of the answer to this question might be that a given discrete or continuous geometry contains a given amount of information represented by its entropy. This entropy might be computed analytically if the geometry is represented by a function, or otherwise might be computed numerically [2]. The complexity of navigating the consciousness from an awareness of one truth to an awareness of another 
would be expected to bear some relationship to the complexity of the truth observed in the initial awareness and the complexity of truth observed in the final awareness. In any case, this methodology of Human-Centric Functional Modeling [3] is designed to maximize our ability to navigate complexity in those systems by allowing us to understand those systems through looking inwards to our experience of our own human system, whether looking inwards to our mind which can by definition be understood, or by looking inward to our consciousness which by definition can be experienced. In the case of the "awareness space" that represents the behavior of the consciousness we can experience, this ability to look inward to analogies with our cognition is critical since only behavior in the conceptual space can be inherently conceptualized and therefore be rationally understood. Gaining an understanding of the functions of any system requires the behavior of that system to be conceptualizable or requires an analogy to exist between that system and one that is conceptualizable. The importance of making this analogy with cognition as a system whose functional states and behavior in moving between those functional states can inherently be conceptualized, is that it allows us to hypothesize mathematical definitions of complexity and other experientially observable properties of other systems such as consciousness, or even of the physical universe as a system, where each hypothesized property is derived from its similarity (in the graph of functional state space) with some property of the cognitive system.

The most important hole in this HCFM methodology is in confirming this hypothesized regarding how it might be used to help navigate mathematical complexity. The broader importance of HCFM suggested by some specific examples in other domains is that through enabling systems to be understood by looking inwards to the nature of human cognition, Human-Centric Functional Modeling is a form of biomimicry that can be used to represent biological systems like our own cognition in a way that enables it to be seen that nature has already solved problems that can be represented in these abstract functional state spaces as the same general problem that must be solved to address problems in a wide range of other systems, including existential challenges from poverty to climate change, where nature has demonstrated those solutions to have worked for hundreds of millions of years [6].

In summary, in Human-Centric Functional Modeling consciousness is represented as moving through a functional state space in which each functional state is an awareness. This functional state space (the space of awarenesses or the "awareness space") is described by a graph in which each node represents an awareness, and in which each node is connected by edges representing the awareness processes through which the consciousness transitions from one awareness to another. Assuming each awareness is associated with a truth, then the potential representation of truth as a geometry suggests truth has an extent. Instead of ending with an argument, this comment concludes with some questions. What then is the maximum size of a truth that can fit within a given awareness? What is the maximum velocity with which awareness can flow from one truth to another? These questions require answering the question of "what do distances in awareness space mean"? They also require answering the question of "what does the size of a truth mean"? Defining distances in awareness space or conceptual space is one of the remaining problems in representing conceptual space, since the understanding of distances in conceptual space is important to applying patterns of biomimicry that might be used to exponentially increase our collective capacity to solve the complexity of the wicked problems facing us today like poverty and climate change [6]. Resolving this question of distances in functional state space in turn might require an understanding of the relationship between truth and geometry.

\section{References}

[1] Ioannis K. Argyros, Santhosh George, On the complexity of extending the convergence region for Traub's method, Journal of Complexity, Volume 56, 2020, 101423, ISSN 0885-064X, https://doi.org/10.1016/j.jco.2019.101423. 
[2] Zenil, H. A Review of Methods for Estimating Algorithmic Complexity: Options, Challenges, and New Directions. Entropy 2020, 22, 612. https://doi.org/10.3390/e22060612

[3] Williams, A. E. (2021). Human-Centric Functional Modeling and the Unification of Systems Thinking Approaches: A Short Communication. Journal of Systems Thinking, 1(1), 5. https://doi.org/10.54120/jost.v1i1.1369 (Original work published August 20, 2021)

[4] C. E. Shannon, “A Mathematical theory of Communication,” Proc. IRE, vol. 27, pp. 379-423, 1948. [5] Murawski, Roman. "Proof Truth in Mathematics" Studia Humana, vol.9, no.3-4, 2020, pp.10-18. https://doi.org/10.2478/sh-2020-0025

[6] Williams, A.E. Are wicked problems a lack of general collective intelligence?. AI \& Soc (2021). https://doi.org/10.1007/s00146-021-01297-8

[7] Newman MEJ (2010) Networks: an introduction. Oxford University Press, New York

[8] Andy E. Williams, A Revolution in Systems Thinking?, Proceedings of the World Organization of Systems and Cybernetics (WOSC) 2021 Congress, Accepted (2021), link 